\title{
3D-printing: an emerging and a revolutionary technology in pharmaceuticals
}

\author{
Gautam SINGHVI 1, Shalini PATIL 1, Vishal GIRDHAR 1, \\ Dinesh K. CHELLAPPAN ${ }^{2}$, Gaurav GUPTA ${ }^{2} *$, Kamal DUA 4, 5, 6
}

${ }^{1}$ Department of Pharmacy, Birla Institute of Technology \& Science (BITS), Pilani, India; ${ }^{2}$ Department of Life Sciences, School of Pharmacy, International Medical University, Kuala Lumpur, Malaysia; ${ }^{3}$ School of Pharmaceutical Sciences, Jaipur National University, Jagatpura, India; ${ }^{4}$ Discipline of Pharmacy, Graduate School of Health, University of Technology Sydney, Ultimo, Australia; ${ }^{5}$ School of Biomedical Sciences and Pharmacy, The University of Newcastle, Callaghan, Australia; ${ }^{6}$ Priority Research Centre for Healthy Lungs, Hunter Medical Research Institute, Lot 1 Kookaburra Circuit, New Lambton Heights, Newcastle, Australia

*Corresponding author: Gaurav Gupta, School of Pharmaceutical Sciences, Jaipur National University, Jagatpura, 302017 Jaipur, India. E-mail: gauravpharma25@gmail.com

\section{A B S T R A C T}

One of the novel and progressive technology employed in pharmaceutical manufacturing, design of medical device and tissue engineering is three-dimensional (3D) printing. 3D printing technologies provide great advantages in 3D scaffolds fabrication over traditional methods in the control of pore size, porosity, and interconnectivity. Various techniques of 3D-printing include powder bed fusion, fused deposition modeling, binder deposition, inkjet printing, photopolymerization and many others which are still evolving. 3D-printing technique been employed in developing immediate release products, various systems to deliver multiple release modalities etc. 3D printing has opened the door for new generation of customized drug delivery with built-in flexibility for safer and effective therapy. Our mini-review provides a quick snapshot on an overview of $3 \mathrm{D}$ printing, various techniques employed, applications and its advancements in pharmaceutical sciences.

(Cite this article as: Singhvi G, Patil S, Girdhar V, Chellappan DK, Gupta G, Dua K. 3D-printing: an emerging and a revolutionary technology in pharmaceuticals. Panminerva Med 2018;60:000-000. DOI: 10.23736/S0031-0808.18.03467-5)

KEY WORDS: Printing, three-dimensional - Drug delivery systems - Tissue engineering.

$\mathrm{C}$ urrently, one of the novel and progressive technology employed in pharmaceutical manufacturing, design of medical device and tissue engineering is three-dimensional (3D) printing. It follows the principles of additive manufacturing wherein layer-by-layer production is done in two dimensional (2D)-fashion to develop a 3D structure based on digital design. Developing a pharmaceutical product by 3D-printing involves few basic common steps like: 1) developing a design of the final product; 2) converting this design into a machine-readable format; 3 ) processing the raw material; and 4) finally printing it followed by postprocessing steps if required. ${ }^{3}$ D printing technologies provide great advantages in $3 \mathrm{D}$ scaffolds fabrication over traditional methods in the control of pore size, porosity, and interconnectivity. The combination of $3 \mathrm{D}$ print- ing technologies and drug delivery systems suggests the excellent possibilities in tissue formation. ${ }^{2}$

\section{Approaches employed for 3D-printing}

Various techniques of 3D-printing are available including powder bed fusion, fused deposition modeling, binder deposition, inkjet printing, photopolymerization and many others which are still evolving. But, amongst all such techniques, very few are utilized in pharmaceutical production. In 2015, powder bed fusion technique was used for processing of the first USFDA approved 3D-printed fast-dissolving tablet (printlet), Spritam ${ }^{\circledR}$ (Aprecia Pharmaceuticals, Blue Ash, OH, USA). ${ }^{3}$ This technique basically involved fusion of high melting point particles with 
low melting point binders. But, despite the successful launch of the first product, there were certain drawbacks associated with powder bed fusion technique such as poor mechanical properties and surface imperfections. Considering such drawbacks, researchers started working on semi-solid extrusion 3D-printing technique, wherein the semi-solid form of raw materials is passed through syringe head to develop bilayered tablets, polypills, etc. Due to the associated disadvantages like requirement of postoperative drying, high friability tablets etc., this technology also doesn't sustain longer. ${ }^{4}$

Fused filament fabrication (FFF) method of 3D-printing overcomes the limitations associated with earlier mentioned technologies and provided certain advantages such as ready-to-use product with high mechanical strength and high resolution. This process is widely known with its trademark name called Fused Deposition Modeling (FDM). The FDM technique involves utilization of hot melt extrusion (HME) technique to develop thermoplastic filaments (mainly of polymer), which are then fed into the 3D printer to develop a 3D product with layer-by-layer deposition technique. HME makes it easy to load the drug onto the filament, which instead includes impregnating drug on the commercially available filaments which is a slow and cumbersome process. ${ }^{4,} 5$ Zhang et. al. prepared aceclofenac loaded tablets using FDM technology in combination with HME technology and studied the impact of different grades of polymers in different ratio on the release rate of drug. ${ }^{6}$

Despite its wide-scale applications, FFF also encompasses drawbacks, one of which is a narrow range of polymers which can be used as carriers for drugs in 3Dprinting. Additionally, these pharmaceutical grade polymers lack mechanical properties, flexibility, and thermal stability which are essential components in processing by 3D-printing technology. Apart from this, another important drawback includes improper miscibility of the drug in the polymer, which is due to semi-crystalline nature of the polymer used. To overcome some of these limitations, nowadays some immiscible fillers and plasticizers (Eudragit ${ }^{\circledR}$, PEG-400, Evonik Health Care, Essen, Germany) are incorporated into the $3 \mathrm{D}$-printed products. However, the ratio of polymer to filler used needs to be optimized to obtain a quality $3 \mathrm{D}$-print. ${ }^{7}$

Meanwhile, many different techniques were developed in order to fabricate $3 \mathrm{D}$ printed macro and micro scale systems, which could improve drug loading, give controlled release and many other advantages. And this was only possible by using various thermoplastic polymers, hydrogels, powders, etc. ${ }^{8}$ With respect to development of 3D printed controlled release systems, certain multilayered bone implants, formulations using porous paper substrates, etc were also developed using 3D printing technology. ${ }^{6}$

Inkjet technique for 3D printing involves the spraying of molten mass which solidifies drop-by-drop to develop final 3D-structure. In photopolymerization 3D printing technique (also known as stereolithography), liquid resins are exposed to high energy light source which can induce polymerization into the resin. In case of Pen-based 3D-printing, it utilizes extrusion principle where the extrude layer-by-layer develops a final 3D product. Newer advanced techniques are also evolved such as racetrack printing, contour crafting, etc. They are providing various advantages such as printing relatively flat products in less time, self-supportive products and a product with a smoother surface.

\section{Applications of 3D-printing technique}

With increasing advancements in technologies, product profile has also improved. Initially, the 3D-printing technique was used to develop immediate release products, but the porous structure which is developed by this technique that retards the release of the drug provided scientist with a path to work on modified release products. Also, researchers are currently working on incorporating gradient of excipients to control the release; utilization of osmotic delivery system in 3D printed products to deliver multiple release modalities; modulating system in such a way as to achieve structured break down of the solid oral dosage form in order to deposit different parts in different regions of lumen, etc.

3D-printing of pharmaceutical products provides advantages of developing complex products with accuracy because it has completed digital control over the printing process. It also provides the major benefit of personalization of medicaments, wherein the amount of drug can be tailored as per the requirement, making it more patient compliant and finally improving the treatment regimen. Additionally, 3D-printing technology is cheap and less time to consume as compared to the conventional techniques which provide an advantage of on-demand manufacturing in some medical emergency situations. ${ }^{1,9}$

3D-printing has been utilized for preparation of various pharmaceutical products such as fast-disintegrating tablets, multi-layered caplets, microneedles, implants and many others. Currently, scientists are even working on merging two vast technologies, nanotechnology and 3D- 
printing technology. Wherein various different nanoformulations (for example nanocapsules) are being loaded on to 3D-printed dosage forms to utilize the benefits associated with nanotechnology such as increased bioavailability, targeting, etc. with 3D-printing such as personalization of medicaments. The idea of combining the benefits of these two technologies has just emerged and there is still a need for research in this area. ${ }^{10}$

\section{Advancements with 3D-printing technology}

Other recent advancements in 3D-printing also involve utilization of Quality-By-Design (QBD) spaces and Process Analytical Technology (PAT) in processing through 3D-printing technology. The availability of various models thus makes it possible to recognize critical process parameters (CPP), predicting intermediate states of raw material processing, simulating the transfer of heat in the process, etc. These things can make the process of 3Dprinting even more controlled in real time (Figure 1).

With increasing advancements in 3D-printing, 3Dprinting is also being used to develop 3D scaffolds which could be utilized for transplantation, 3D-bioprinting thus involves layer-by-layer positioning of biological materials, living cells with high order of mechanical control to place these elements properly in order to fabricate 3D biological structures. This technology is surely providing us with 3D-printed tissues, organs, etc. but their utilization in human transplantation is still a matter of socio-ethical concern. Nevertheless, it can be definitely used for laboratory testing in cosmetology, toxicology, etc. ${ }^{11,12}$

3D-printing technology is widely being used to produce complex and personalized medicines on demand. Researchers are coming up with many newer products and even innovations in technologies to broaden the scope of production. There are huge research and commercialization opportunities in $3 \mathrm{D}$ printing technique. Some manufacturing challenges are also associated with $3 \mathrm{D}$ printing

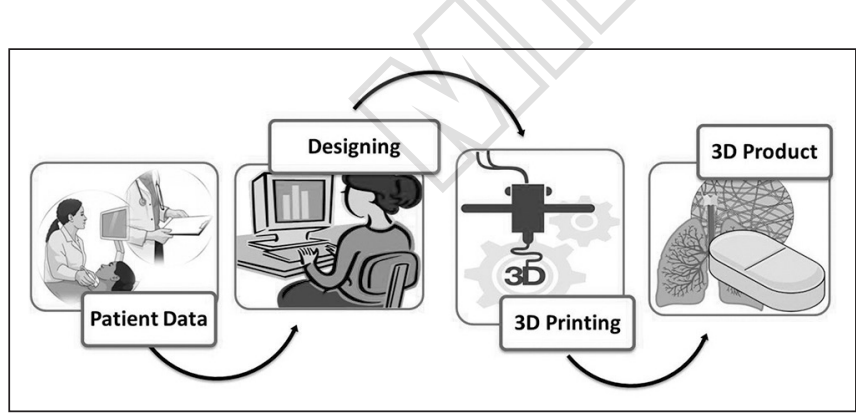

Figure 1.-Application of 3D printing. including banding, residuals, warping, leaning, etc. To overcome these issues associated with the 3D-printing technology of pharmaceuticals, there is need to improve existing processing equipment of 3D-printing. ${ }^{1}$ The fusion of advanced drug delivery using 3D printing technology with conventional pharmaceutical technologies can be anticipated to utilize the advantages of $3 \mathrm{D}$ printing with proven effectiveness of conventional technologies for the development of customized, precise and cost-effective drug delivery systems.

But, as 3D-printed pharmaceuticals are patentable in nature, their regulatory control also plays an important role. The FDA is currently approving 3D printed medical devices through the $510(\mathrm{k})$ regulatory process. In 2016, a draft guidance has been issued by USFDA on the Technical Considerations for Additive Manufactured Devices for the manufacturer of 3D printing-based devices. However, it is still questionable whether FDA will consider just the 3Dprinted product or the 3D-printer or both of them. Thus, the approval of first 3D-printed pharmaceutical product does not assure that next upcoming products will also be approved. ${ }^{13,14}$ For effective product evaluation and approval, regulatory agencies are working on to understand the effect of $3 \mathrm{D}$ geometric design, material attributes, and $3 \mathrm{D}$ printing process parameters on the performance of $3 \mathrm{D}$ printed solid dosage forms. Regulatory bodies also felt the need of mechanistic models for 3D printing processes which can be utilized as predictive tool for product performance in different disease and patient conditions. It is expected that current research on $3 \mathrm{D}$ printing with regulatory requirement can bring the most effective and safer pharmaceutical product. ${ }^{14}$

\section{Conclusions}

$3 \mathrm{D}$ printing has opened the door for new generation of customized drug delivery with built-in flexibility for safer and effective therapy. This new tool is gaining enormous attention which is not only restricted to pharmaceuticals in designing customized drug delivery systems but also expanding its horizons in the area of tissue engineering etc. Overall, this technology is bringing advancements and revolution in the area of pharmaceutical and biological sciences.

\section{References}

1. Norman J, Madurawe RD, Moore CM, Khan MA, Khairuzzaman A. A new chapter in pharmaceutical manufacturing: $3 \mathrm{D}$-printed drug products. Adv Drug Deliv Rev 2017;108:39-50. 
2. Lee JW, Cho DW 3rd. 3D Printing technology over a drug delivery for tissue engineering. Curr Pharm Des 2015;21:1606-17.

3. Solanki NG, Tahsin M, Shah AV, Serajuddin AT. Formulation of 3D Printed Tablet for Rapid Drug Release by Fused Deposition Modeling: Screening Polymers for Drug Release, Drug-Polymer Miscibility and Printability. J Pharm Sci 2018;107:390-401.

4. Sadia M, Sośnicka A, Arafat B, Isreb A, Ahmed W, Kelarakis A, et al. Adaptation of pharmaceutical excipients to FDM 3D printing for the fabrication of patient-tailored immediate release tablets. Int J Pharm 2016;513:659-68.

5. Goyanes A, Kobayashi M, Martínez-Pacheco R, Gaisford S, W. Basit A. Fused-filament 3D printing of drug products: Microstructure analysis and drug release characteristics of PVA-based caplets; 2016

6. Ursan ID, Chiu L, Pierce A. Three-dimensional drug printing: a structured review. J Am Pharm Assoc (2003) 2013;53:136-44.

7. Beck RC, Chaves PS, Goyanes A, Vukosavljevic B, Buanz A, Windbergs M, et al. 3D printed tablets loaded with polymeric nanocapsules: an innovative approach to produce customized drug delivery systems. Int J Pharm 2017;528:268-79.
8. Moulton SE, Wallace GG. 3-dimensional (3D) fabricated polymer based drug delivery systems. J Control Release 2014;193:27-34.

9. Trenfield SJ, Awad A, Goyanes A, Gaisford S, Basit AW. 3D Printing Pharmaceuticals: Drug Development to Frontline Care. Trends Pharmacol Sci 2018;39:440-51

10. Katakam P, Dey B, Assaleh FH, Hwisa NT, Adiki SK, Chandu BR, et al. Top-Down and Bottom-Up Approaches in 3D Printing Technologies for Drug Delivery Challenges. Crit Rev Ther Drug Carrier Syst 2015;32:61-87.

11. Murphy SV, Atala A. 3D bioprinting of tissues and organs. Nat Biotechnol 2014;32:773-85.

12. Munaz A, Vadivelu RK, St. John J, Barton M, Kamble H, Nguyen NT. Three-dimensional printing of biological matters. Journal of Science: Advanced Materials and Devices 2016;1:1-17.

13. Stones JA, Jewell CM. 3D printing of pharmaceuticals: patent and regulatory challenges. Pharm Pat Anal 2017;6:145-9.

14. Ventola CL. Medical Applications for 3D Printing: Current and Projected Uses. P\&T 2014;39:704-11.

Conflicts of interest.-The authors certify that there is no conflict of interest with any financial organization regarding the material discussed in the manuscript. Article first published online: June 1, 2018. - Manuscript accepted: May 22, 2018. - Manuscript received: April 28, 2018. 